\title{
Atypical presentation of carcinoid heart disease (case report and literature's review)
}

azin alizadehasl ${ }^{1}$, Bahar Galeshi $^{1}$, mehdi peighambari ${ }^{1}$, maryam moradian $^{1}$, and $\operatorname{arash}$ hashemi $^{1}$

${ }^{1}$ Affiliation not available

October 19, 2020

\begin{abstract}
Carcinoid disease is a rare neoplasm but it's an important etiology of intrinsic right heart valve disorders that leads to right heart failure. carcinoid heart involvement is a common complication of disease that effects on morbidity and mortality of the patient.
\end{abstract}

key Clinical Message : Carcinoid heart disease is a well-known complication of carcinoid tumor that affects morbidity and mortality of the patients. It may be asymptomatic in the early stages, therefore, it's of a high importance to both consider the abnormality properly and to screen the patients to prevent misdiagnosis.

\section{Introduction:}

Neuroendocrine tumors (NETs) are infrequent neoplasms, commonly occurred in 2.5 to 5 cases of every 100,000 people in a population, and are mostly originated from gastrointestinal tract. (1) The secretion of vasoactive substances by these tumors is the main reason to trigger carcinoid syndrome symptoms. Carcinoid tumors usually develop gradually over years. They frequently show few or no symptoms till they are bulky enough to be symptomatic or have metastasized mostly to the liver. Less common locations for invasion and causing symptoms are skeletal and pulmonary systems. ${ }^{(1,2,3)}$ One of the common complications of carcinoid syndrome is cardiac involvement caused by the direct action of vasoactive substances ${ }^{(3)}$. Although it can occur in up to $60 \%$ of the cases during the course of the disease ${ }^{(2,4)}$, carcinoid heart disease can be the first manifestation in about $20 \%$ of the patients ${ }^{(3,4)}$. As cardiac manifestations related to a poor long-term medical prospect and mortality rate ${ }^{(2)}$ detection of cardiac disease in early stages, on the one hand, have a significant value for us, with the improvement in the field of cardiac surgery, on the other hand, valve replacement can be an alternative opportunity for symptomatic patients. The aforementioned procedure improves the patients' survival rate and enhances their quality of life, only if it is done in the corresponding optimal time. We will review some important practical aspects of the carcinoid syndrome and cardiac involvement by explaining a real case examined in our clinic.

Case presentation:

A 75-year-old woman referred for the first time to our cardio-oncology clinic with a chief complaint of progressive dyspnea, fatigue, peripheral edema, and intermittent palpitation. The study of her documentation in medical records helped us reckon a gastrointestinal neuroendocrine tumor with liver metastasis that she had been undergoing for 4 years. She was receiving medication by her oncologist during those years. The patient was also on cardiac medication like aspirin, atorvastatin, losartan, metohexal, and furosemide with the diagnosis of heart failure but without regular follow-up or any complete orientation about the disease. 
The previous medical history was also remarkable for long-term HTN. She was both ill and weak. Physical examination showed Heart Rate (HR) of approximately 80 beats/min and arterial blood pressure (BP) of about $140 / 75 \mathrm{mmHg}$.

The sounds of breathing were diminished over her right lower lung field. Jugular venous pressure was in normal limit. Cardiac auscultation was revealed normal for the first and the second heart sounds and a grade II/VI systolic ejection was murmuring along the left sternal border. Lower limb edema was apparent without any cyanosis.

However, a transthoracic 2-D echocardiography had been performed in the past, but the result was not available for us to get the related information. Thus, as the first step, a 2-D transthoracic echo was done comprehensively which showed normal LV size as well as mild systolic dysfunction with diastolic dysfunction, severe RA and RV enlargement with RV dysfunction. The tricuspid valve hereby was thickened, retracted and hypomobile resulting in severe free tricuspid regurgitation caused by the lack of leaflet cooptation without any valvular stenosis.

Assessment of the pulmonary valve also showed thickened, malcoapted valve with severe insufficiency, without any stenosis.

Left heart valves were not involved. Although they were mildly thickened, the changes seemed to be senile. According to these findings, carcinoid heart involvement was considered for this patient. Mostly, dyspnea was related to hypertensive cardiomyopathy, diastolic dysfunction, anemia, and low cardiac output which is due to RV failure. Other symptoms can be explained through right heart failure. In order to assess the palpitation, 48 hours of Holter monitoring was done which showed frequent PACs and PVCs. The biochemical test was done, and electrolyte disturbances were corrected. The patient's medications were adjusted according to her tolerance under close monitoring in serial follow-ups. Gradually, her symptoms were improved. It is probable that she could be an example of those patients with cardiac symptoms undergoing mismanaged conditions and being known just as a patient with heart failure, and according to the overall condition and the severity of the disease, it was not advisable for her to undergo a surgery.

Discussion:

Carcinoid disease is a paraneoplastic condition, arising from vasoactive hormones secreted by carcinoid tumors ${ }^{(3)}$. As mentioned earlier, it is probable to discover them anywhere in the body, however, the pulmonary origin is the most common after digestive system (1).

Chiefly, they arise from the small intestine, commonly in the ileum, and release different kinds of vasoactive materials like serotonin, 5-hydroxytryptamine, 5-hydroxytryptophan, histamine, tachykinins, bradykinin, and prostaglandins into the circulatory system that lead to the clinical manifestations of carcinoid syndrome. Approximately, around 30\%-40\% of cases with features of carcinoid syndrome were defined by periods of flushing, bronchospasm, hypotension, and sometimes hypertension, diarrhea, and skin involvement during the course of the disease (3).

Carcinoid tumors usually develop gradually over the years. They frequently show few or no symptoms till they are large enough or have metastasized mostly to the liver. Subsequently, the productions of the tumors cannot be deactivated by the liver and reach the systemic circulation $(1,2)$. Nevertheless, about $5 \%$ of the patients, mainly those with the primary ovarian or pulmonary or even some cases of midgut tumors with retroperitoneal metastases, might show carcinoid symptoms in the absence of liver metastases $(1,5)$. Once large volumes of vasoactive substances reach the right side of the heart, carcinoid heart disease will begin to appear. Generally, the left side of the heart is spared because these substances are metabolized by the lungs. In spite of being rare, left heart involvement is concomitant with Patent Foramen Ovale and primary bronchopulmonary carcinoid disease $(2,3)$. The prevalence of Carcinoid Heart Disease (CHD) that was first described in 1954 (5) is expected to occur in more than $50 \%$ of the affected patients $(1,6)$ or even more, up to $70 \%$ of the cases (5) in different stages of severity. According to the data collection of outsized studies, the highest incidence rate of carcinoid heart disease is in patients with small bowel NETs. The ratio is roughly 
$72 \%$, however, in nearly $18 \%$ of the occasions, the primary location cannot be detected precisely $(1,6)$. A small amount of male predominance $(60 \%)$ has been described, with mean age range of 56 to 63 years at the time of diagnosis (6). The cardiac disease may be detected as an initial manifestation of carcinoid syndrome, a wellknown complication that decreases the patient's survival rate and is related to the major cause of morbidity and mortality in these groups of patients. ${ }^{(6,3,11)}$ The patient's life quality and outcome with carcinoid heart disease will be decreased without therapeutic options, with 3 years of survival of around $31 \%$ compared to $68 \%$ in those patients without CHD. On the contrary, the survival rate of patients with established carcinoid heart disease has been improved over the past few decades, probably due to improvement and advances in diagnostic imaging technologies, novel therapies, cardiac surgery, and better perioperative care ${ }^{(1)}$. The pathogenesis of carcinoid heart disease is complex and not comprehensively recognized. 5-HT receptors play a fundamental role in the pathogenesis of the disease. They are well manifested in the heart, mainly 5-HT2B subtype, located mostly on the cardiac valves. Occurrence of the mitogenic effects on fibroblasts and smooth muscle cells, triggering of inflammatory cytokines, and up-regulation of transforming growth factor-b1 are induced through the activation of 5-HT receptors, all resulting in the deposition of plaquelike substances on the endocardial surfaces of valve leaflets, subvalvular apparatus (chordae and papillary muscles), cardiac chambers, and occasionally, being seated on the intima of the pulmonary arteries and aorta as well as the venae cavae ${ }^{(1)}$. Plaque formation leads to annular restriction, leaflet thickening, and merging of the subvalvular apparatus, as a result, the significant degenerative changes of valvular apparatus lead to severe retraction and noncoaptation of the valve leaflets. The tricuspid valve is mostly affected by the isolated regurgitation, and in smaller amounts by both stenosis and regurgitation. In contrast, the combined effect of regurgitation and stenosis of the pulmonary valve is usually the main manifestation of the disease and, furthermore, the isolated involvement happens less frequently. The increased rate of pulmonary stenosis is likely correlated to its smaller annulus. Isolated regurgitation is usually found in leftsided carcinoid valvular disease. In comparison with rheumatismal valvular disease or valvular involvement in ergot-like substances, right valvular involvement is predominant and is a clinical key to differentiation (8). Shi-Min described the relative proportion of the valve pathologies in CHD by the help of the aforementioned method: $97 \%$ of the patients had tricuspid valve disease Most of them, about 90\%, showed moderate or severe tricuspid insufficiency; smaller ones had concurrent tricuspid stenosis. Pulmonary valve involvement was diagnosed in $88 \%$ of the cases. Accordingly, $81 \%$ of these patients showed pulmonary regurgitation and $53 \%$ had pulmonary stenosis. Only in $7 \%$ of the patients, left-sided disease was detected which was mostly presented by mitral regurgitation with mild-to-moderate severity (7). These structural and valvular lesions ultimately lead to right-sided heart failure, but the history of flushing, diarrhea, and pulmonary symptoms can differentiate CHD from other etiologies that give rise to heart failure. Functional capacity and severity of right ventricular failure were both prominent and fundamental predictors of the outcome in these patients.

Coronary artery vasospasm is also associated with carcinoid HD, usually seen in the patients with a history of nonocclusive coronary artery disease. Generally, according to the endothelial status, serotonin can stimulate vasodilatory or constrictive responses (2). In the field of proven ischemic heart disease, a carcinoid heart can lead to coronary artery vasospasm, angina, and in-stent thrombosis (4).

Arrhythmia s have rarely been reported. The underlying mechanism is thought to increase sympathetic hyperactivity related to vasoactive materials (2). Atrial and ventricular arrhythmia such as atrial fibrillation, ventricular tachycardia, ventricular fibrillation, and cardiac arrest, (4) are also reported. Infrequently, anisolated intra-cardiac mass, estimated in about $4 \%$ of the patients (1), might be a manifestation of carcinoid heart disease without any valvular involvement (4). These well-defined, homogeneous, and noninfiltrative masses are mostly found on both ventricles (including the ventricular septum) during echocardiography. Carcinoid metastasis to cardiac structures can be asymptomatic and be manifested as a solitary mass, or can cause ventricular outflow tract obstruction (2). Heart failure due to pericardial disease and constrictive pericarditis is a rare manifestation of the disease that may be present without any obvious valvular diseases (4).

\section{Clinical Features of CHD Patients}


Clinical manifestations of CHD are often unremarkable in early stages. Patients with tricuspid and pulmonary valve diseases, even in advanced stages of involvement, might be asymptomatic for a long time due to the low pressure of pulmonary circulation. According to the studies, around $57 \%$ of the patients with moderate to severe tricuspid insufficiency are either asymptomatic or have only mild symptoms $(2,3$, 9 ), therefore, the detection of carcinoid heart involvement might be postponed without echocardiographic screening. As a result, highly suspicious and regular follow-up is needed to establish the timely diagnosis $(2,9)$.

Primary symptoms usually consist of exertional fatigue and dyspnea. Concurrently, with tumor progression and increased levels of serotonin, progressive right-sided heart failure will appear and the patient's symptoms will exacerbate (9).

The main findings on physical examinations include edema and elevated jugular venous pressure. With severe tricuspid insufficiency, the "V" wave may be prominent in the jugular venous pulse. A palpable right ventricular impulse may be detected. Murmurs of tricuspid and pulmonic valve regurgitation may be audible. Auscultatory findings are mostly negligible due to the low-pressure resistance in the right circulation. A systolic murmur of pulmonic stenosis may be heard. In the rare examples of left-sided disease, murmurs of mitral and aortic regurgitation may be detected. Patients may show central cyanosis in the presence of right to left shunt $(4,8)$.

Imaging : 2D echocardiography is the modality of choice in the primary evaluation of patients with carcinoid syndrome. It revealed thickened and retracted valve leaflets in a semi-open position with reduced mobility, annular constriction, thickening, and fusion of the subvalvular apparatus and finally regurgitation and /or stenosis in various degrees $(1,7,8,9)$. Severe tricuspid regurgitation resulted in an RV volume overload and RV/RA dilatation (6). Severe tricuspid insufficiency classically showed a characteristic 'dagger-shaped' spectrum on the Doppler profile (early peak pressure increases with a subsequent rapid drop) (9). Synchronously, the pulmonic valve disease could also occur to a lesser extent, $49 \%$ to $69 \%$ of the patients (6). In a study that reviewed echocardiographic findings of 74 patients with CHD; the patients showed tricuspid and pulmonary regurgitation rates of $100 \%$ and $81 \%$, respectively. Moreover, pulmonic stenosis affected $53 \%$ of the cases. Subsequently, $7 \%$ of the patients were noted to have left-sided involvement, mostly due to the presence of PFO $(87 \%)$ or primary lung neuroendocrine tumor $(13 \%)$. Besides giving rise to valvular involvement, CHD can disturb RV strain regardless of the severity of the valvular disease, therefore, it could be considered as a sensitive marker to detect the disease in early stages. Global LV strain is also reduced slightly. In rare cases, neuroendocrine tumors can metastasize directly to the myocardium. Larger masses could be visualized by 2D echocardiography, but masses with the size of less than $1 \mathrm{~cm}$ might be difficult to identify by 2D TTE. In comparison with 2D TTE, 3D TTE provides some more prominent advantages. It allows for a detailed assessment of valvular involvement as well as the surrounding structures and a more accurate evaluation of myocardial mass in cases of direct metastasis (10). According to a determinant statement by the American College of Cardiology, regarding the diagnosis and management of CHD, an echocardiogram should be obtained in all patients with carcinoid syndrome and high suspicion for CHD. In patients with established CHD, an echocardiogram should be repeated every 3-6 months or during alterations in clinical status.

In contrast, the European Neuroendocrine Tumor Society (ENETS) guidelines recommend performing TTE annually among patients with known CHD $(3,10)$. Although $2 \mathrm{D}$ echocardiography has distinct advantages like availability, fairly low cost, and lack of radiation, it's not free of limitations. Other modalities like cardiac MRI or CT may be helpful in these fields(figure1) (10).

Although overt CHD is diagnosed easily by $2 \mathrm{D}$ echocardiography, detection of the early stages of the disease may be challenging. As the severity of cardiac involvement has an obvious effect on a patient's survival, early detection and treatment strategies are the most important goals that will improve clinical outcome $(10,11)$.

Utilization of sensitive and specific biochemical markers that are associated with the presence and severity of cardiac involvement may lead to the more appropriate use of echocardiography as a screening tool. Some biomarkers are useful in this field. N-Terminal pro-Brain Natriuretic Peptide (NT-proBNP) has significantly 
been escalated in patients with CHD and is correlated with disease progression, symptomatic status, and overall survival. Evaluation of NT-proBNP with a cut-off level of $260 \mathrm{pg} / \mathrm{ml}$ is recommended as a screening biomarker with sensitivity and specificity of $92 \%$ and $91 \%$, respectively. Negative and positive predictive values are $98 \%$ and $71 \%$.

Plasma or urinary level of 5-Hydroxyindoleacetic Acid (5-HIAA) "a metabolite of serotonin" is higher in cardiac involvement and the increased level of this metabolite, above $300 \mathrm{mmol} / 24 \mathrm{~h}$, is related to the increment of the risk of evolvement or progression of carcinoid heart disease by a factor of 2-3 times $(1,11)$.

As mentioned above, life expectancy will decrease with cardiac disease occurrence, therefore, early detection and treatment may prevent right heart failure and improve overall survival. The management of these patients is complex and needs a multidisciplinary approach and close collaboration between medical oncologists, expert cardiologists in the field of CHD, and cardiac surgeons. The final goal is to postpone disease progression. As the rate of progression isn't predictable, regular follow-up and intensive monitoring is also required to detect cardiac involvement in the early stages and to determine the timing of surgery before the occurrence of the advanced disease $(7,8,11)$.

Treatment of the cardiac abnormalities in carcinoid syndrome is based on the management of volume status and heart failure symptoms as well as treatment of NET itself and the reduction in the production of the related hormones, and finally heart valve surgery (12). Volume management is necessary and diuretics (the most commonly used diuretic is loop diuretic and in necessity, it's applied with aldosterone antagonist or thiazide) together with fluid and salt restriction had better be advised $(4,13)$. However, caution should be taken to avoid intravascular depletion in these patient groups, regardless of the severity of RV dilation or failure, because it could result in decreased cardiac output and would make them prone to the symptoms of low cardiac output like lightheadedness, syncope, and fatigue. Other conventional heart failure medications like beta-blocker, angiotensin-converting enzyme inhibitors, and angiotensin receptor blockers may be prescribed in some cases, but the efficacy of those therapies is unknown.

All of these medications are palliatives and relieve symptoms of patients. As a result, they can't prevent the progression of the underlying carcinoid syndrome or cardiac involvement (4). The pharmacological treatment of cardiac disease is largely related to the management of vasoactive hormones level by somatostatin analogues. The aforementioned groups of medications like octreotide and lanreotide, are somatostatinreceptor inhibitors. Accordingly, they decrease the secretion of serotonin and its metabolites like 5-HIAA, and have a great importance in controlling the symptoms. It's necessary to stabilize the patients by using the aforementioned medications with adequate symptom control prior to any procedure to reduce the risk of the complications. Although these medications show significant symptomatic benefit, they have not been demonstrated to be useful in controlling carcinoid syndrome or reversing the established cardiac disease, however, they could be referred to only slow down the progression. Having said that significant effects on overall survival have not been proven yet.

For patients' refractory to somatostatin analogues, the new strategy includes the use of everolimus, interferonalpha, peptide receptor radionuclide therapy, and telotristat etiprate which have been showing promising results $(2,4,8,11)$.

The use of cytotoxic thermotherapy in refractory cases may be linked to an augmented risk of progressive carcinoid heart disease which is due to the burst serotonin release via destruction of the cells. Other medical therapies including Bosentan, a dual endothelin receptor antagonist, may take part as an effective method to prevent the expansion of valvular and mural fibrosis (8). Surgical resection of the primary tumor and hepatic metastases seem to decrease the risk of cardiac progression although the metastatic diseases can be a contraindication for surgery. Hepatic surgery in a patient with established cardiac involvement conveys a prominent risk of life-threatening periprocedural bleeding due to elevated transhepatic and portal pressure secondary to severe tricuspid regurgitation. Therefore, valve surgery should be recommended prior to hepatic intervention and, subsequently, hepatic resection can be performed relatively safe with a similar outcome.

In more extensive diseases, hepatic intra-arterial therapies like embolization can be performed alternatively, 
but the efficacy of these kinds of therapies on disease progression is limited (8.11). Surgical intervention is the only established effective therapy in cardiac heart diseases in comparison with medical therapy alone with long-term survival. Advances in cardiac surgical and anesthetic management have resulted in obvious improvement of the surgical outcome and have reduced the perioperative complications and mortality rate over time. Due to the benefits of the former surgeries in these groups of the patients, the effective management of CHD patients is to undergo valve surgery in optimal time by the help of experienced surgeons and their colleagues to minimize the adverse effects and improve quality of life. Appropriate timing of a surgical intervention is not clear. There are different scoring systems, mostly according to tricuspid involvement, but generally, according to the current recommendations, valve surgery should be considered if the patient is severely symptomatic or when the severe valvular disease is established $(4,8,11)$, or even when progressive asymptomatic RV dysfunction and dilatation are present. However, surgery is recommended in some groups of patients with controlled baseline carcinoid tumor with at least 12 months of anticipated postoperative survivals from NET disease (6). Limited evidence is available in favor of surgery in asymptomatic patients. Clearly, in the asymptomatic patients, the results of these interventions haven't been proven yet $(8,11)$. Møller and colleagues reported a higher perioperative survival rate in early intervention in asymptomatic or mildly symptomatic patients when compared to severely symptomatic patients (14).

Valve replacement is more common. Due to the extensive fibrotic changes, repairing is not possible in most cases. In stenotic lesions that are not candidates for valve surgery, balloon valvuloplasty is an alternative approach, however, short-term hemodynamic and functional benefits along with the relapse of the disease have limited the value of this procedure. A more controversial topic in the field of valve surgery is the selection of valve prosthesis. On the one hand, there is a concern about bioprosthesis valve degeneration $(8,9)$, even in early stages as early as 3 months after implantation. Due to the high levels of intractable vasoactive products, carcinoid plaque reformation would be induced $(1,9)$. On the other hand, the use of mechanical prosthetic valve needs to be lifelong anticoagulation therapy in the patients that are prone to bleeding due to hepatic dysfunction along with an increased risk of valve thrombosis whenever they are a candidate for further surgical intervention $(1,6,8)$ The decision-making on the type of valve prosthesis is complex and is better to be individualized based on the patient risk assessment. The advantages and disadvantages summarized in Table 1 should be explained in detail with the patients as a part of the decision-making process. New generations of bioprosthesis valve with more durability may provide hope for more successful rates of valve surgery and increase new trends in the fields of treatment with less recurrence. Postoperative management should continue controlling hormone levels to prevent the recurrence of the disease (9). Patent Foramen Ovale should be closed in the time of valve surgery (6).

Conclusion :

Carcinoid syndrome appeared as a paraneoplastic symptom of rare NETs but was a well-recognized complication of the syndrome; cardiac involvement has a significant effect on morbidity and mortality of the patients. Right heart valves are more involved. Echocardiography is recommended for the evaluation of patients and, subsequently, the echo parameters in conjunction with other modalities and laboratory tests can aid to determine the optimal time for surgery. CS and CHD need a multidisciplinary approach for treatment.

\section{Author contribution:}

- Dr Alizadehasl. A, Dr Galeshi.B \& Dr Peighambari.M: study conception and design,

- Dr Alizadehasl. A, Dr Galeshi.B: literature review and manuscript writing

- All authors discussed and interpreted data and commented on the manuscript

Acknowledgement statement:

Not applicable.

Conflict of interes:

no conflict of interest. 
Refrence:

1. Davar J, Connolly HM, Caplin ME, Pavel M, Zacks J, Bhattacharyya S, Cuthbertson DJ, Dobson R, Grozinsky-Glasberg S, Steeds RP, Dreyfus G. Diagnosing and managing carcinoid heart disease in patients with neuroendocrine tumors: an expert statement. Journal of the American College of Cardiology. 2017 Mar 6;69(10):1288-304.

2. Ram P, Penalver JL, Lo KB, Rangaswami J, Pressman GS. Carcinoid heart disease: review of current knowledge. Texas Heart Institute Journal. 2019 Feb;46(1):21-7.

3. Khay K, Arous S, Bentaoune T, Drighil A, Habbal R. Carcinoid heart disease revealed by cyanosis with both right and left valvular involvement: a case report. Journal of medical case reports. 2018 Dec 1;12(1):23.

4. Hassan SA, Palaskas NL, Agha AM, Iliescu C, Lopez-Mattei J, Chen C, Zheng H, Yusuf SW. Carcinoid heart disease: a comprehensive review. Current Cardiology Reports. 2019 Nov 1;21(11):140.

5. Bhattacharyya S, Davar J, Dreyfus G, Caplin ME. Carcinoid heart disease. Circulation. 2007 Dec 11;116(24):2860-5.

6. Bertin N, Favretto S, Pelizzo F, Mos L, Pertoldi F, Vriz O. Carcinoid heart disease: starting from heart failure. Journal of investigative medicine high impact case reports. 2017 Jun;5(2).

7. Yuan SM. Valvular disorders in carcinoid heart disease. Brazilian journal of cardiovascular surgery. 2016 Oct;31(5):400-5.

8. Luis SA, Pellikka PA. Carcinoid heart disease: diagnosis and management. Best Practice \& Research Clinical Endocrinology \& Metabolism. 2016 Jan 1;30(1):149-58.

9. Grozinsky-Glasberg S, Grossman AB, Gross DJ. Carcinoid heart disease: from pathophysiology to treatment- 'Something in the way it moves'. Neuroendocrinology. 2015;101(4):263-73.

10. Agha AM, Lopez-Mattei J, Donisan T, Balanescu D, Iliescu CA, Banchs J, Kim PY, Palaskas NL, Yusuf S, Gladish G, Hassan S. Multimodality imaging in carcinoid heart disease. Open heart. 2019 Jun 1;6(1): e001060.

11. . Hart EA, Meijs TA, Meijer RC, Dreijerink KM, Tesselaar ME, de Groot CA, Valk GD, Chamuleau SA. Carcinoid heart disease: a guide for screening and timing of surgical intervention. Netherlands Heart Journal. 2017 Sep 1;25(9):471-8.

12. . Rubin de Celis Ferrari AC, Glasberg J, Riechelmann RP. Carcinoid syndrome: update on the pathophysiology and treatment. Clinics. 2018;73.

13. . Lichtenauer M, Pichler T, Eder S, Mirna M, Magnes T, Wernly B, Paar V, Jung C, Prinz E, Seitelberger R, Hoppe UC. Carcinoid heart disease involving the left heart: a case report and biomarker analysis. ESC heart failure. 2019 Feb;6(1):222-7.

14. . Møller JE, Pellikka PA, Bernheim AM, Schaff HV, Rubin J, Connolly HM. Prognosis of carcinoid heart disease: analysis of 200 cases over two decades. Circulation. 2005 Nov 22;112(21):3320-7.

15. . Alizadeh-Sani Z, Vakili-Zarch A, Kiavar M, Bahadorian B, Nabavi A. Eosinophilic endomyocardial fibrosis and strongyloides stercoralis: a case report. Res Cardiovasc Med. 2013 May 1;2(2):104-5.

16. . Hakim HA, Samadikhah JA, Alizadehasl AZ, Azarfarin RA. Chronobiological rhythms in onset of massive pulmonary embolism in Iranian population. Middle East J Anesthesiol. 2009 Oct; 20:369-75.

17. . Sadeghpour A, Alizadehasl A. The right ventricle: a comprehensive review from anatomy, physiology, and mechanics to hemodynamic, functional, and imaging evaluation. Arch Cardiovasc Imaging. 2015 Nov 1;3(4): e35717.

Supplementary Data :

Figure 1:carcinoid heart disease

\section{Hosted file}

Untitledssss-1.pdf available at https://authorea.com/users/318927/articles/487434-atypicalpresentation-of-carcinoid-heart-disease-case-report-and-literature-s-review 